\title{
Discrete simulation of model, loose cohesive powders: plastic consolidation, fractal microstructure and tensile strength
}

Cite as: AIP Conference Proceedings 1145, 889 (2009); https://doi.org/10.1063/1.3180073

Published Online: 01 July 2009

Francisco Gilabert, Jean-Noël Roux, and Antonio Castellanos

ARTICLES YOU MAY BE INTERESTED IN

Compressive consolidation of strongly aggregated particle gels

Journal of Rheology 57, 1347 (2013); https://doi.org/10.1122/1.4817436

Challenge us.

What are your needs for periodic signal detection?

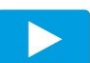

Watch

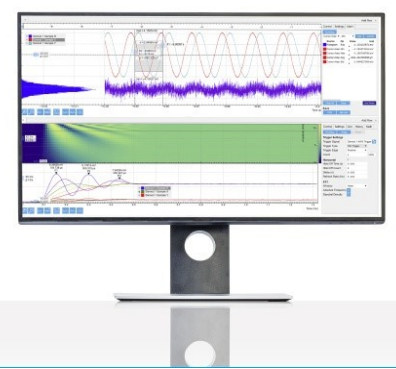

- Instruments 


\title{
Discrete simulation of model, loose cohesive powders: plastic consolidation, fractal microstructure and tensile strength
}

\author{
Francisco Gilabert* ${ }^{*}$ Jean-Noël Roux ${ }^{\dagger}$ and Antonio Castellanos* \\ ${ }^{*}$ Universidad de Sevilla, Facultad de Física, Avenida Reina Mercedes s/n, 41012 Sevilla, Spain \\ ${ }^{\dagger}$ Université Paris-Est, Laboratoire Navier, 2 allée Kepler, Cité Descartes, 77420 Champs-sur-Marne, France
}

\begin{abstract}
Isotropic packings of cohesive disks in 2D are studied by discrete, stress-controlled numerical simulations. Depending on the assembling process and on whether contacts possess rolling resistance (RR), configurations form under low pressure $P$ with varying solid fraction $\Phi$ and fractal dimension $d_{F}$ describing small scale correlations below some blob size $\xi$. The gradual collapse observed under growing $P$ is described as a linear relation between $\ln P$ and $1 / \Phi$ within some range, once the influence of initial conditions has faded out, and until a maximum density is approached. This corresponds to a decrease of $\xi$ that is compatible with the fractal blob model. The isotropic tensile strength is always considerably smaller than the naive Rumpf estimate, and grows with consolidation. Coordination numbers in systems with small RR change little while density increases by large amounts in consolidation.
\end{abstract}

Keywords: cohesive granular material; rolling resistance; fractal; colloid aggregation; consolidation; plasticity index; strength PACS: $81.05 . \mathrm{Rm}, 81.20 . \mathrm{Ev}, 83.80 . \mathrm{Kn}, 81.40 . \mathrm{Lm}$

\section{INTRODUCTION}

Cohesive granular systems, such as some soil materials [1] and powders [2], can form very loose structures, which undergo considerable plastic compaction under growing stress intensity $[1,3]$ and can resist tension. The present communication investigates those phenomena by discrete element simulations in quasistatic conditions. Such a situation, with loose systems, has hardly been addressed by numerical means, as the recent literature rather explored dynamic compaction $[4,5]$, gravity deposition [6], steady flows [7], or denser materials [8, 9]. We introduce a simple model material and report on its consolidation properties, in relation to its microstructure, thus presenting a brief account of studies published in two recent papers $[10,11]$, to which a section with new results on the resistance to tension is added. The paper ends with a short discussion.

\section{MATERIAL AND INTERACTION LAWS}

Just like in Refs. [10, 11], which might be consulted for more details on the model, we consider 2D assemblies of $N$ disks, with diameters uniformly distributed in the interval $[a / 2, a]$. The disks interact with their contacting or very close neighbors, with forces that combine normal and tangential elasticity (involving spring constants $K_{N}, K_{T}$ ), Coulomb friction (with friction coefficient $\mu$ ), some viscous damping (the values of which are irrelevant in quasistatic conditions), and some simplified form of van der Waals attraction. The latter consists in an attrac- tive force which vanishes beyond the value $D_{0}=a / 1000$ of the gap $h$ separating neighbors, varies linearly with $h$ to reach its maximum intensity $F_{0}$ for $h=0$ and stays constant for $h<0$, i.e., when the particles are in contact. We also introduce rolling resistance (RR) in some samples, hence a rolling friction parameter (a length) $\mu_{R}$ such that the local rolling moment $\Gamma$ at a contact satisfies $|\Gamma| \leq \mu_{R} F_{N}^{e}$, with $F_{N}^{e}$ the repulsive elastic part of normal force $F_{N}$. RR is physically attributed to small asperities and modeled similarly to standard sliding friction [10], whence a rolling stiffness constant $K_{R}$. For contact deflection $-h_{0}=F_{0} / K_{N}$, while the static normal force $-K_{N} h+F_{0}$ vanishes, the maximum tangential force is $\mu F_{0}$ and the maximum rolling moment is $\mu_{R} F_{0}$.

Table 1 lists the values of micromechanical parameters used for most simulations, in dimensionless form.

TABLE 1. Values of interaction parameters.

\begin{tabular}{cccccc}
\hline$\mu$ & $\frac{a K_{N}}{F_{0}}$ & $\frac{K_{T}}{K_{N}}$ & $\frac{D_{0}}{a}$ & $\frac{K_{R}}{K_{N} a^{2}}$ & $\frac{\mu_{R}}{a}$ \\
\hline 0.5 & $10^{5}$ & 1 & $10^{-3}$ & $10^{-4}$ & 0 or 0.005 \\
\hline
\end{tabular}

\section{INITIAL CONFIGURATIONS}

In an initial stage, a granular gas is generated with solid fraction $\Phi_{I}$ and a Maxwell velocity distribution, corresponding to mean quadratic velocity $V_{0}$ for particles of diameter $a$ and mass $m$. $V_{0}$ should be compared to the value $V^{*}=\sqrt{2 F_{0} D_{0}}$ of the receding velocity in a contact that is necessary to overcome attraction. While the disks collide and stick to one another, in a process akin 
to isotropic ballistic aggregation, $\Phi$ is kept constant, until all particles form one unique cluster. Then, a nonvanishing pressure $P$ is applied to the periodic rectangular simulation cell, the edge lengths of which vary according to an adapted Parrinello-Rahman scheme [10]. The strain rate $\dot{\varepsilon}$ is constrained by condition $\dot{\varepsilon} T_{0}<0.05$, involving characteristic time $T_{0}=\sqrt{\mathrm{am} / F_{0}}$. The dimensionless ratio $P^{*}=a P / F_{0}$ conveniently expresses the competition of confining pressure with contact adhesion. This procedure is applied to 5 samples of $N=1400$ disks, 4 with $N=5600$ and 1 with $N=10976$, with the parameters of Table 1 , and $\Phi_{I}=0.36$. The application of the first nonzero confining pressure, $P_{I}^{*}=0.01$, is regarded as part of the assembling process. It entails a substantial density increase, to $\Phi=0.524 \pm 0.008$ with $\mathrm{RR}$ and to $\Phi=0.472 \pm 0.008$ without RR. One such configuration is shown in Fig. 1. Coordination number $z$ is about 3.1

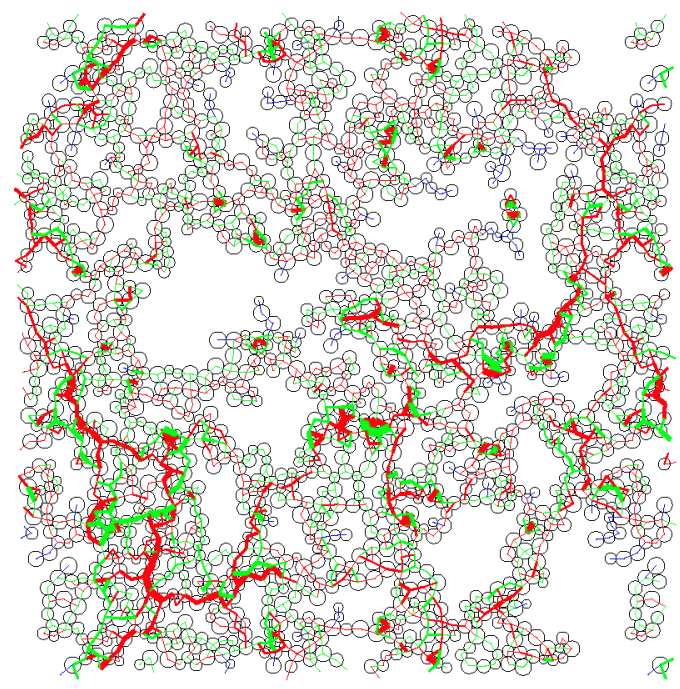

FIGURE 1. One configuration $(N=1400)$ with RR under $P^{*}=0.01$. Compressive forces are shown in red, tensile ones in green, line thickness encoding intensity. A few contacts (in blue) bear vanishing forces $\left(h=h_{0}\right)$.

without RR and 2.9 with $\mathrm{RR}$ in such states. It decreases to nearly 2 if a large value $\mu_{R} / a=0.5$ is adopted.

\section{PLASTIC CONSOLIDATION}

The pressure is then stepwise increased, from cohesiondominated states at small $P^{*}$ to confinement-dominated ones at large $P^{*}$. In each step, $P^{*}$ is multiplied by a constant factor $10^{1 / 8}$, and one waits for equilibrium to be satisfied with good accuracy: the net force - and moment - on each particle is lower than $10^{-4} F_{1}$ - resp. $10^{-4} F_{1} a-$ with $F_{1}=\operatorname{Max}\left\{F_{0}, a P\right\}$, with a similar tolerance on controlled internal stresses. The stability of such equilibrium states can also be checked with stiffness matrcies [11]. Considerable structural changes are witnessed under growing $P^{*}$, as the initial loose structure (Fig. 1) gradually collapses and loses its porosity. The compaction is stopped at $P^{*}=13.3$, above which no significant further compaction is observed. The sample structure and force distribution is then similar to that of a cohesionless system, as apparent in Fig. 2. The evolution

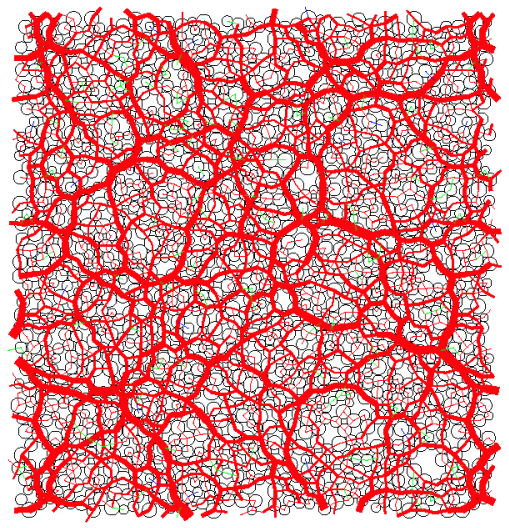

FIGURE 2. Configuration of Fig 1 , compacted to $P^{*}=13.3$.

of density with pressure during compaction is shown in Fig. 3. No size dependence is observed and the data of all samples are averaged over. Conforming to the literature, in which the void index $e=-1+1 / \Phi$ is used as a state variable, such consolidation curves can be represented as $1 / \Phi$ versus $\ln P^{*}$. They are quite sensitive to a small RR, and can be modeled as follows, introducing three regimes for growing $P^{*}$. In the first one, for $P^{*}$ of the order of $P_{I}^{*}$, the first applied nonvanishing pressure, the initial structure resists the pressure increase, and $\Phi$ stays constant. Then, in regime II, pressure increments cause irreversible compaction and a straight line is ap-

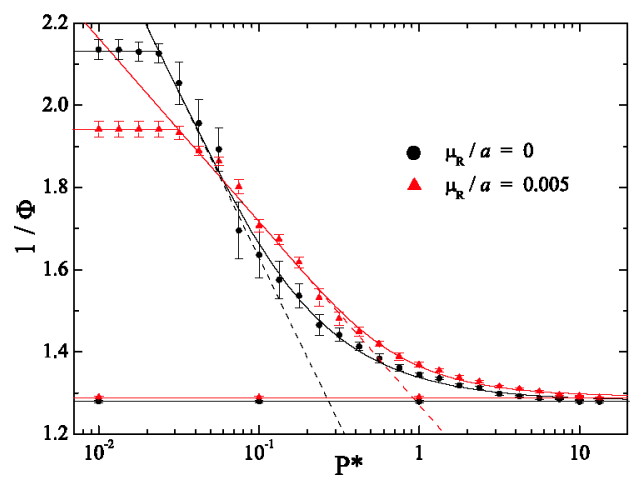

FIGURE 3. Consolidation data and fit to Eq. (3), for systems with (red triangles) and without (black dots) a small RR. Dashed lines: fits to Eq. (1). Continuous lines: fits to Eq. (3) and (bottom) decompression from highest $P^{*}$.

proached as $N \rightarrow \infty$. This is the classical form of consolidation curves, defining a plasticity index $\lambda$ :

$$
\frac{1}{\Phi}=\frac{1}{\Phi_{0}}-\lambda \ln \frac{P^{*}}{P_{0}^{*}} .
$$


In Eq. (1), values $\Phi_{0}$ and $P_{0}^{*}$ correspond to the end of regime $I$, and depend on the initial assembling method. However, we could check [11] that various factors affecting $\Phi_{0}$ and $P_{0}^{*}$, such as $\Phi_{I}$ or $V_{0} / V^{*}$, become irrelevant once regime II is reached. The consolidation behavior expressed by Eq. 1 is thus intrinsic, independent on the initial state. Finally, a maximum solid fraction $\Phi_{\max }$ is approached at large $P^{*}$, as a power law,

$$
\frac{1}{\Phi}=\frac{1}{\Phi_{\max }}+\frac{A}{\left(P^{*}\right)^{\alpha}}
$$

A possible complete formula interpolating between Eqs. 1 and 2 is (defining a crossover pressure $P_{1}^{*}$ by setting $\Phi=\Phi_{\max }$ in (1))

$$
\frac{1}{\Phi}=\frac{1}{\Phi_{0}}-\lambda \ln \left\{\frac{P^{*}}{P_{0}^{*}}\left[1-\exp \left(-\left[\frac{P_{1}^{*}}{P^{*}}\right]^{\alpha}\right)\right]^{1 / \alpha}\right\}
$$

which is found (see Fig. 3) to fit the data appropriately. Eq. (3) requests $A=\lambda\left(P_{1}^{*}\right)^{\alpha} /(2 \alpha)$ in (2). Table 2 lists the values of fitting parameters.

TABLE 2. Values of parameters $\lambda, \Phi_{\max }$ and $\alpha$ used to fit the consolidation curve with parameters of Table 1 , and in a sample with larger RR, with Eq. (3). Corresponding $P_{1}^{*}$ values are $0.271 \pm 0.033,0.900 \pm 0.064$ and $2.6 \pm 0.4$.

\begin{tabular}{|c|ccccc|}
\hline$\mu_{R} / a$ & $P_{0}^{*}$ & $\Phi_{0}$ & $\lambda$ & $\Phi_{\max }$ & $\alpha$ \\
\hline 0 & 0.0237 & 0.469 & 0.349 & 0.7808 & $0.91 \pm 0.10$ \\
0.005 & 0.0316 & 0.515 & 0.194 & 0.7745 & $1.08 \pm 0.16$ \\
0.5 & 0.0178 & 0.382 & 0.25 & 0.724 & $0.86 \pm 0.24$ \\
\hline
\end{tabular}

On reducing $P^{*}$ at any stage (as shown on Fig. 3 for $\left.P^{*}=13.3\right), \Phi$ remains constant, apart from small, nearly elastic [11] changes. Subsequently, plastic compaction is resumed only if the highest value $P_{c}^{*}$ applied in the past, the preconsolidation pressure, is exceeded.

\section{DENSITY CORRELATIONS}

Open, ramified structures such as the one of Fig. 1 are frequent in colloid aggregation processes, and exhibit a self-similar structure characterized by a fractal dimension $d_{F}$. Ideal aggregation processes (e.g., diffusionlimited, reaction-limited or ballistic) have been studied a lot [12] and lead to different values of $d_{F}$ [13]. Since the density of a fractal object vanishes in the thermodynamic limit, arbitrarily large systems of finite solid fraction $\Phi$ only display a fractal structure below some correlation length or "blob size" $\xi$, which satisfies (in 2D) [12]

$$
\xi \propto \Phi^{-1 /\left(2-d_{F}\right)}
$$

In the traditional models, aggregates are rigid, undeformable and unbreakable objects, whence a loopless structure and coordination number $z=2$. We are dealing here with a more realistic version of ballistic aggregation, with a complete mechanical model for particle interactions. The result of the ideal aggregation process with rigid clusters is only approached for large RR with, then, $z \rightarrow 2$ at small $P^{*}$ (or, also, in the limit of $V_{0} / V^{*} \rightarrow 0$ under $P^{*}=0[10]$, as initial clusters are hardly disturbed as aggregation proceeds).

$d_{F}$ is measured on computing the Fourier transform of the density autocorrelation function (or "scattering intensity"), $I(k)$, which should be approximately constant for wavevector $k$ below $2 \pi / \xi$ and decrease as $k^{-d_{F}}$ in the fractal range $2 \pi / a \gg k \gg 2 \pi / \xi$. Fig. 4 , in spite of

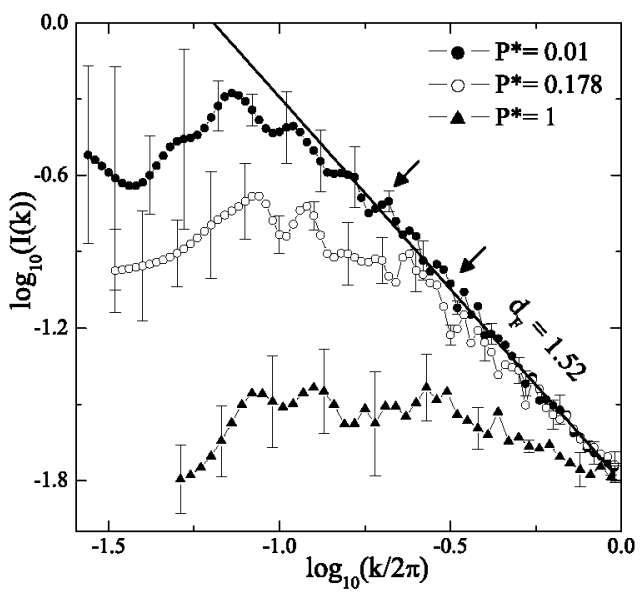

FIGURE 4. Scattering intensity per unit area versus wave vector $k$ in systems with RR for 3 values of $P_{c}^{*}$.

large error bars at small $k$, shows that our results abide by such expectations. Correlations coincide for large $k$ for different $P^{*}$, indicating that consolidation essentially affects the large scale features of the microstructure. The slope of $I(k)$ versus $k$ on the logarithmic plot yields $d_{F}=1.52 \pm 0.04$, which coincides with the fractal dimension ( $1.55 \pm 0.02)$ obtained in ideal ballistic aggregation [13], despite a different connectivity: $z$ is well above 2 , it increases with $P_{c}^{*}$ from about 2.9 to 3.1. Correlation length $\xi$ can be estimated from the abscissa of the intersection of the initial plateau with the line of slope $-d_{F}$. $\xi$ decreases as $P^{*}$ increases until all signatures of a fractal microstructure vanish (for $P_{c}^{*} \sim 1$ ). Due to the limited fractal range and corrections to scaling, relation (4) cannot be accurately checked, although it correctly describes [10] the change of $\xi$ between $P_{c}^{*}=0(\Phi=0.36$, $\xi / a \simeq 9.5)$ and $P_{c}^{*}=0.01(\Phi=0.515, \xi / a \simeq 5.0)$.

Interestingly, the same data analysis in systems without RR yields quite a different value of $d_{F}$, near 1.9: a small level of $\operatorname{RR}\left(\mu_{R} / a=0.005\right)$ has a large effect on the fractal dimension. 


\section{TENSILE STRENGTH}

Some values of tensile strength, as measured under isotropic stresses (i.e., a negative value $P_{T}^{*}$ of $P^{*}$ ), are shown in Fig. 5, as a function of preconsolidation pressure $P_{c}^{*} . P_{T}^{*}$ might be measured either with a stepwise loading (as used in compression), or on smoothly stretching the specimen until the inner tension reaches a maximum. Both procedures agree. Remarkably, $P_{T}^{*}$ turns out

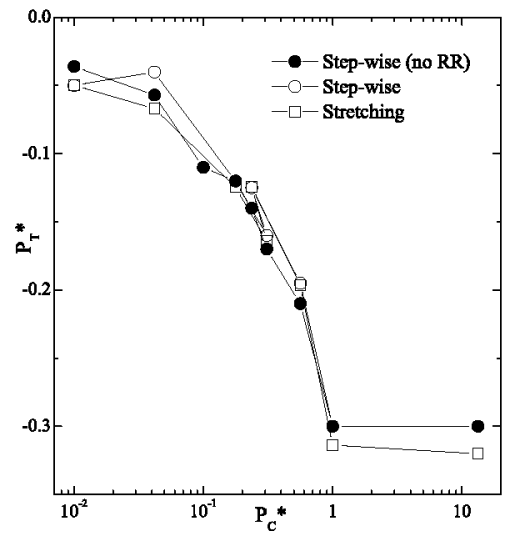

FIGURE 5. Isotropic tensile strength versus $P_{c}^{*}$ in a sample with $N=1400$.

to be very similar for small $R R$ or without $R R$. This contrasts with the sensitivity of consolidation behavior - in which the system gets continuously broken and repaired - to a small RR level. $P_{T}^{*}$ is reached for a very small strain level $\left(<10^{-4}\right)$, irrespective of $P_{c}^{*}$. The rupture can thus be regarded as brittle - although for low $P_{c}^{*}$ samples may still withstand smaller tensions, as they get progressively torn apart in a strain-controlled test. Classically, the strength of a cohesive material is assessed with the so-called Rumpf formula [9], which amounts in the present case to replacing the average normal force $F_{N}$ by $-F_{0}$ in relation $F_{N}=7 \pi a P /(z \Phi)$ (which is nearly exact [10] and directly stems from the classical expression of stresses as sums over contacts involving forces and branch vectors). The Rumpf formula was proposed decades ago [14], based on the assumption that a large population of contacts simultaneously break in tension. It ignores the specific disorder and microstructure of cohesive packings and overestimates the tensile strength by a large factor $\left(\simeq 3\right.$ for large $P^{*}$, above 50 for $\left.P^{*}=0.01\right)$. On the other hand, a Rumpf-like formula can be tried [2] at scale $\xi$, based on a description of the system as a packing of blobs joined by fragile bonds with tensile strength $\sim F_{0}$. This provides a rough estimate of $P_{T}^{*}$, up to some unknown numerical factor, and predicts $P_{T}^{*} \propto \xi^{-1}$. The variations of $P_{T}^{*}$ (Fig. 5) are compatible with such a relation, as it increases by a factor of about 6 while $\xi$ decreases in the same proportion.

\section{CONCLUSIONS}

We have retrieved the classical consolidation behavior of cohesive granular assemblies and powders with a simple model. The study of the mechanical properties of loose cohesive granular assemblies can benefit from the concepts developed and applied in the fields of gels or colloidal aggregates (fractal dimension, blob size). Thus estimates for such quantities as tensile strength, on accounting for the characteristic blob size, are improved over simple, particle-level approaches. However, the scaling regime is not necessarily well developed, and the mechanical behavior depends on connectivity properties beyond the simplest fractal characteristics. Although consolidation regime II is observed to coincide with the self-similar scaling regime for $\xi$, a prediction of plasticity index - associated with complex rearrangement events - is still unavailable. Another clear conclusion is the importance of rolling friction in contacts (even a small $\mu_{R}$ changes the geometric and mechanical properties). Our observations call for both more detailed investigations of rupture and plasticity mechanisms, for varying RR levels, and more general classifications, in 2 and 3 dimensions, of the rich variety of structures appearing in loose cohesive systems (would mechanical properties change along with $d_{F}$ with diffusion-limited instead of ballistic aggregation ?)

\section{REFERENCES}

1. J. K. Mitchell, Fundamentals of soil behavior, Wiley, New York, 1993.

2. A. Castellanos, Advances in Physics 54, 263-376 (2005).

3. J. M. Valverde, A. Ramos, A. Castellanos, and P. Watson, Powder Technology 97, 237-245 (1998).

4. D. E. Wolf, T. Unger, D. Kadau, and L. Brendel, "Compaction of cohesive powders," in Powders and Grains 2005, edited by R. García Rojo, H. J. Herrmann, and S. McNamara, Balkema, Lisse, 2005, pp. 525-533.

5. G. Bartels, T. Unger, D. Kadau, D. E. Wolf, and J. Kertész, Granular Matter 7, 139-143 (2005).

6. K. J. Dong, R. Y. Yang, R. P. Zou, and A. B. Yu, Phys. Rev. Lett. 96, 145505 (2006).

7. P. G. Rognon, J.-N. Roux, M. Naaïm, and F. Chevoir, $J$. Fluid Mech. 596, 21-47 (2008).

8. S. Luding, Powder Technology 158, 45-50 (2005).

9. V. Richefeu, M. S. El Youssoufi, and F. Radjai, Phys. Rev. E 73 (2006).

10. F. A. Gilabert, J.-N. Roux, and A. Castellanos, Phys. Rev. E 75, 011303 (2007).

11. F. A. Gilabert, J.-N. Roux, and A. Castellanos, Phys. Rev. E 78, 031305 (2008).

12. P. Meakin, J. Sol-Gel Sci. Tech. 15, 97-117 (1999).

13. B. Smimov, Phys. Rep. 188, 1-78 (1990).

14. H. Rumpf, Chem. Eng. Tech. 30, 144 (1958). 